\title{
Orbital angular momentum due to modes interference
}

\author{
IRving RONDÓN OJEDA ${ }^{1 *}$, FrANCISCO SOTO-EGUIBAR $^{2}$ \\ ${ }^{1}$ School of Computational Sciences, Korea Institute for Advanced Study, KIAS, \\ 85 Hoegiro, Seoul 02455, Republic of Korea \\ ${ }^{2}$ Instituto Nacional de Astrofisica Optica y Electronica, INAOE, \\ Luis Enrique Erro 1, Santa María Tonantzintla, Puebla, México 72840 \\ ${ }^{*}$ Corresponding author: irondon@kias.re.kr
}

\begin{abstract}
We present generalized expressions to calculate the orbital angular momentum for invariant beams using scalars potentials. The solutions can be separated into transversal electric TE, transversal magnetic TM and transversal electromagnetic TE/TM polarization modes. We show that the superposition of non-paraxial vectorial beams with axial symmetry can provide a well-defined orbital angular momentum and that the modes superposition affects the angular momentum flux density. The results are illustrated and analyzed for Bessel beams.
\end{abstract}

Keywords: invariant beams, orbital angular momentum, mode interference, scalar potentials.

\section{Introduction}

Since the pioneering and interesting work of the orbital angular momentum presented by ALLEN et al. [1], the study of the subject has covered many interesting applications [2]; a compilation overview over the last 25 years, with several theoretical and experimental applications was reported in [ $\underline{3}]$. The authors in [4] have recently demonstrated that a rotationally arranged nano-antenna can be used to convert the phase information in a twisted light beam into spectral information, which hence can be used to classify the phase state of the twisted light beam. This effect has a strong influence on the optical properties of dielectric and plasmonic particles and it is useful for new technological applications. The physical properties of the orbital angular momentum have the potential to improve the performance of optical communication systems in different ways [ [ $]$ ], but it is not the aim of this manuscript to explore the full list of possibilities. Exploiting the physical properties of the orbital angular momentum is the subject of an increasing number of research topics nowadays. In this context, here we focus on the use of the scalar formalism for studying the orbital angular momentum for invariant beams; due to its simplicity, this approach has been theoretically $[\underline{6}]$ and experimentally $[\underline{7}, \underline{8}]$ used and tested. 
Recently, the scalar representation has been used to describe scattering problems using partial waves series in the far field approximation, where the impinging source is a structured field [ $\underline{9}-\underline{12}]$. Effects also considering the polarization [13], the extinction [14] and driven acoustic radiation forces [15] have also been considered. Furthermore, the scalar approach is a natural representation for the structured beams family, also known as "non-diffracting beams". The study of these fields covers different areas, from quantum mechanics to astrophysics [16]. Such fields are the Bessel [17], Mathieu [18] and

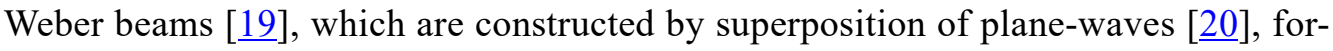
malism which is called angular spectrum representation [21]. These ideal fields propagate indefinitely without changing their transverse intensity distribution [22], even in the presence of massive phase perturbations and into inhomogeneous media [23]. As an interesting application, among others, this physical effect increases the resolution and contrast to image sub-cellular components and organelles in different microscopy methods [24].

Otherwise, the study of structured invariant beams is of increasing interest in optical physics. Their properties make them particularly attractive for optical design [르] , for studying propagation through etalons and crystals [26]. Another interesting example is the use of Bessel beams for driving an optimal single tractor beam for dielectric particles with cylindrical shape [27].

The study of non-paraxial orbital angular momentum was recently revisited in [28] using Bessel beams; in that work, the authors show well defined orbital angular momentum properties, without considering the possibility of contributions due to mixed modes superimposed along their propagation. To the best of our knowledge, this feature has not been fully explored. However, recently, using the scalar potential approach, fundamentals electromagnetic properties, such as the energy density, the Poynting vector, the Maxwell stress tensor for non-paraxial beams were derived [29]. Currently, the study of the Poynting vector has drawn considerable interest due to its features and properties along the propagation.

In the context of structured beams, Novitsky [30] has shown that Bessel beams possess negative values in the longitudinal and azimuthal components of the Poynting vector, which depends on the superposition of mixed modes and on the phase detuning between the complex amplitudes $c_{\mathrm{TE}}$ and $c_{\mathrm{TM}}$ of the transversal electric or magnetic part of the beam representation. Since then, many interesting results have been reported, such as the explanation of optical pulling forces [31], effects in metamaterials [32], tractor beams [33] and optical manipulation [34], to name a few of them. Another interesting case was reported for $\mathrm{X}$-waves in [35], where the propagation direction of their negative Poynting vector could be locally changed using carefully chosen complex amplitudes; however, we showed [29] that the negative behavior can be found independently of the mode interference. Nonetheless, the Poynting vector behavior presented in the Bessel beams and in the X-waves, mentioned above, has opened a discussion related to tractor beams generation, and with other interesting applications, such as the forces that can be locally oriented in a direction opposite to the propagation wave vector [흐]. 
Then, what is the relationship between mixed modes and the negative Poynting vector (NPV)? This effect can be physically defined as an uncommon response produced by the local sign change in the Poynting vector components along its propagation. Recently, we reported in [37] to deepen the understanding of the NPV to study the invariant family beams. The main result of our work was to show the negative local change of the negative Poynting vector, which can be independently obtained without the superposition of mixed modes TE/TM, as it is in the case of Weber beams [37]. Additionally, considerable interest in theoretical and experimental studies of the vectorial structured fields is driven by the possibility to create a wide variety of exotic optical focal fields with homogeneous and spatially inhomogeneous states of polarization; an interesting review of the wide scope of interest and applications is presented in [효] .

In this context, the study of the properties of the orbital angular momentum from the theoretical an experimental points of view can open new engineering technologies. Using the concept of the Poynting vector can be useful, as shown by proposals to measure the orbital angular momentum using the superposition of vector mixed modes TE/TM. This was performed and tested for Laguerre-Gauss beams [39]. This approach may open interest for other structured fields, as it was also proposed by using $\mathrm{X}$ waves in ultrashort optical pulses [40]. Even, in the case of two electromagnetic plane waves with the same angular frequency and different wave vectors, the superposition fields reveal highly nontrivial structure in the local momentum and spin densities [41], that can be used to enhance the optical manipulations of small particles. For example, the spinning dynamics can be driven by superposing two vortex beams with respective circular and radial polarizations such that the particle spins around a certain optical axis [42]. The authors in $[43,44]$ have pointed out the importance of the Poynting vector for obtaining orbital angular momentum from spatial superposition of the Poynting vector beams.

This article is organized as follows: in Section 2, we briefly review the theoretical framework based on the scalar potential approach; in Section 3, we present the general negative Poynting vector for the whole invariant beams family; in Section 4, we study the orbital angular momentum in their transversal and longitudinal propagation due to mixed modes and in Section 5, we present our conclusions.

\section{Maxwell equations in terms of scalar potentials}

Following the formalism proposed by STRATTON [ㄴ5], we write the electromagnetic fields as

$$
\begin{aligned}
& \mathbf{E}=c_{\mathrm{TE}} \mathbf{M}(\mathbf{r})+c_{\mathrm{TM}} \mathbf{N}(\mathbf{r}) \\
& \mathbf{H}=-i \sqrt{\frac{\varepsilon}{\mu}}\left[c_{\mathrm{TE}} \mathbf{N}(\mathbf{r})+c_{\mathrm{TM}} \mathbf{M}(\mathbf{r})\right]
\end{aligned}
$$

where $\mathbf{M}(\mathbf{r})$ and $\mathbf{N}(\mathbf{r})$ are vector fields proposed as

$$
\mathbf{M}(\mathbf{r})=\nabla \times[\hat{\mathbf{a}} \psi(\mathbf{r})]
$$




$$
\mathbf{N}(\mathbf{r})=\frac{1}{k} \nabla \times \mathbf{M}(\mathbf{r})
$$

being $\psi$ a scalar field, $\hat{\mathbf{a}}$ an arbitrary unit vector that determines the direction of propagation (which we will choose as the $Z$ axis, so $\hat{\mathbf{a}}=\hat{\mathbf{e}}_{3}$ ), $k$ the magnitude of the wave vector, $\varepsilon$ the electric permittivity, $\mu$ the magnetic permeability, and $c_{\mathrm{TE}}$ and $c_{\mathrm{TM}}$ two arbitrary complex numbers. This approach has been successfully used to study the properties of the family of invariant beams, theoretically and experimentally [ $\underline{6}-\underline{8}]$.

It is straightforward to verify that if a scalar field $\psi(\mathbf{r})$ satisfies the Helmholtz equation

$$
\nabla^{2} \psi+k^{2} \psi=0
$$

then the fields (1a) and (1b) satisfy the Maxwell equations; so, the scalar field $\psi(\mathbf{r})$ will be named scalar potential. Note that the vector fields, $\mathbf{M}$ and $\mathbf{N}$, are orthogonal, that is $\mathbf{M} \cdot \mathbf{N}=0$, and solenoidal, i.e. $\nabla \cdot \mathbf{M}=0$ and $\nabla \cdot \mathbf{N}=0$.

On the other hand, for any invariant beam, the spatial evolution of the scalar potential $\psi$ can be described by its transverse and longitudinal parts [녀]. The transverse part $\varphi\left(u_{1}, u_{2}\right)$ will depend only on the transverse coordinates, $u_{1}, u_{2}$, and the longitudinal part $Z(z)$ will depend on the longitudinal coordinate $z$ (as we selected $\hat{\mathbf{a}}=\hat{\mathbf{e}}_{3}$ ), physically the propagation axis; i.e., we can write

$$
\psi\left(u_{1}, u_{2}, z\right)=\varphi\left(u_{1}, u_{2}\right) Z(z)
$$

After substituting (5) in the Helmholtz equation, we easily obtain that $\varphi\left(u_{1}, u_{2}\right)$ satisfies the two dimensional transverse Helmholtz equation

$$
\nabla_{\mathrm{T}}^{2} \varphi+k_{\mathrm{T}}^{2} \varphi=0
$$

where $\nabla_{\mathrm{T}}^{2}$ is the Laplacian transversal operator, which has a specific form in each coordinate system, and the longitudinal part is $Z(z)=\exp \left(i k_{z} z\right)$ with the dispersion relation $k^{2}=k_{\mathrm{T}}^{2}+k_{z}^{2}$. The two dimensional transverse Helmholtz equation (6) can be separated in Cartesian, cylindrical, parabolic cylindrical and elliptical cylindrical coordinates [46], and that gives origin to plane waves, Bessel beams, Weber beams and Mathieu beams, respectively. Then, we can write the vector operator fields, Eqs. (2) and (3), as follows:

$$
\mathbf{M}=-\exp \left(i k_{z} z\right) \nabla_{\mathrm{T}}^{\perp} \varphi
$$

where

$$
\nabla_{\mathrm{T}}^{\perp}=-\hat{\mathbf{e}}_{1} \frac{1}{h_{2}} \frac{\partial}{\partial u_{2}}+\hat{\mathbf{e}}_{2} \frac{1}{h_{1}} \frac{\partial}{\partial u_{1}}
$$

and $\hat{\mathbf{e}}_{1}$ and $\hat{\mathbf{e}}_{2}$ are the base unit vectors corresponding to the transversal direction, and $h_{1}$ and $h_{2}$ are the corresponding scale factors. We note that in the four coordinate sys- 
tems, in which Eq. (6) can be separated, the scale factor $h_{3}$ is equal to 1 . It is also easy to verify that

$$
\mathbf{N}=\frac{\exp \left(i k_{z} z\right)}{k}\left(i k_{z} \nabla_{\mathrm{T}}+\hat{\mathbf{e}}_{3} k_{\mathrm{T}}^{2}\right) \varphi
$$

where $\nabla_{\mathrm{T}}$ is the transversal part of the $\nabla$ operators, i.e.

$$
\nabla_{\mathrm{T}}=\hat{\mathbf{e}}_{1} \frac{1}{h_{1}} \frac{\partial}{\partial u_{1}}+\hat{\mathbf{e}}_{2} \frac{1}{h_{2}} \frac{\partial}{\partial u_{2}}
$$

It is worth to notice that the transversal vector operators are related as $\nabla_{\mathrm{T}}^{\perp}=\hat{\mathbf{e}}_{3} \times \nabla_{\mathrm{T}}$.

\section{A generalized Poynting vector for scalars potentials}

The Poynting vector represents the directional power flux per unit area of an electromagnetic field. For harmonic electromagnetic fields, the time average of the Poynting vector is given by [47]

$$
\langle\mathbf{S}\rangle=\frac{1}{2} \operatorname{Re}\left(\mathbf{E} \times \mathbf{H}^{*}\right)
$$

Substituting expressions (1a) and (1b) for the electromagnetic fields into (11), a generalized expression for the Poynting vector of any invariant beam is obtained [29],

$$
\langle\mathbf{S}\rangle=\left|c_{\mathrm{TE}}\right|^{2}\left\langle\mathbf{S}_{\mathrm{TE}}\right\rangle+\left|c_{\mathrm{TM}}\right|^{2}\left\langle\mathbf{S}_{\mathrm{TM}}\right\rangle+\left\langle\mathbf{S}_{\mathrm{TE} / \mathrm{TM}}\right\rangle
$$

where

$$
\left\langle\mathbf{S}_{\mathrm{TE}}\right\rangle=\frac{1}{2 k} \sqrt{\frac{\varepsilon}{\mu}} \operatorname{Re}\left[\left(\nabla_{\mathrm{T}} \varphi \cdot \nabla_{\mathrm{T}} \varphi^{*}\right) k_{z} \hat{\mathbf{e}}_{3}-i k_{\mathrm{T}}^{2} \varphi^{*} \nabla_{\mathrm{T}} \varphi\right]
$$

is the transversal electric part,

$$
\left\langle\mathbf{S}_{\mathrm{TM}}\right\rangle=\frac{1}{2 k} \sqrt{\frac{\varepsilon}{\mu}} \operatorname{Re}\left[\left(\nabla_{\mathrm{T}} \varphi \cdot \nabla_{\mathrm{T}} \varphi^{*}\right) k_{z} \hat{\mathbf{e}}_{3}+i k_{\mathrm{T}}^{2} \varphi^{*} \nabla_{\mathrm{T}} \varphi\right]
$$

is the transversal magnetic part, and the interference modes TE/TM

$$
\begin{aligned}
\left\langle\mathbf{S}_{\mathrm{TE} / \mathrm{TM}}\right\rangle=\frac{1}{2 k^{2}} \sqrt{\frac{\varepsilon}{\mu}} \operatorname{Re}[ & i\left(c_{\mathrm{TE}} c_{\mathrm{TM}}^{*} k^{2}+c_{\mathrm{TE}}^{*} c_{\mathrm{TM}} k_{z}^{2}\right)\left(\nabla_{\mathrm{T}} \varphi^{*} \times \nabla_{\mathrm{T}} \varphi\right) \cdot \hat{\mathbf{e}}_{3} \\
+ & \left.c_{\mathrm{TE}}^{*} c_{\mathrm{TM}} k_{z} k_{\mathrm{T}}^{2} \hat{\mathbf{e}}_{3} \times \nabla_{\mathrm{T}}\left(\varphi^{*} \varphi\right)\right]
\end{aligned}
$$

The time averaged Poynting vector is independent of the $z$ coordinate and it satisfies $\nabla \cdot\langle\mathbf{S}\rangle=0$, as was proven in [48]. It is important to remark that the interference term in the Poynting vector, expressed in Eq. (15), is different from zero for any in- 
variant beam, of course, whenever at least one of the two constants $c_{\mathrm{TE}}$ and $c_{\mathrm{TM}}$ is not null. Physically this confirms the negative behavior trough the propagation; these results were recently reported in [37] using the Weber beams. The study of its effect on the orbital angular momentum is done in the following sections.

\section{Orbital angular momentum density}

For harmonic electromagnetic fields, the time averaged linear momentum per unit volume carried is $\langle\mathbf{p}\rangle=\langle\mathbf{S}\rangle / c^{2}[\underline{44}, \underline{49}]$, and then, the time averaged orbital angular momentum density is $\langle\mathbf{j}\rangle=\mathbf{r} \times\langle\mathbf{p}\rangle=\mathbf{r} \times\langle\mathbf{S}\rangle / c^{2}$; using Eqs. (13), (14) and (15), we can find explicitly the contributions from the transverse electric, from the transverse magnetic and from the transverse electric/transverse magnetic TE/TM mixed modes. The transversal electric TE part is

$$
\left\langle\mathbf{j}^{\mathrm{TE}}\right\rangle=\frac{\varepsilon}{2 \omega}\left|c_{\mathrm{TE}}\right|^{2} \operatorname{Re}\left[k_{z}\left(\nabla_{\mathrm{T}} \varphi \cdot \nabla_{\mathrm{T}} \varphi^{*}\right) \mathbf{r} \times \hat{\mathbf{e}}_{3}-i k_{\mathrm{T}}^{2} \varphi^{*} \mathbf{r} \times \nabla_{\mathrm{T}} \varphi\right]
$$

whereas the transversal magnetic TM contribution has the form

$$
\left\langle\mathbf{j}^{\mathrm{TM}}\right\rangle=\frac{\varepsilon}{2 \omega}\left|c_{\mathrm{TM}}\right|^{2} \operatorname{Re}\left[k_{z}\left(\nabla_{\mathrm{T}} \varphi \cdot \nabla_{\mathrm{T}} \varphi^{*}\right) \mathbf{r} \times \hat{\mathbf{e}}_{3}+i k_{\mathrm{T}}^{2} \varphi \mathbf{r} \times \nabla_{\mathrm{T}} \varphi^{*}\right]
$$

and the interference mixed modes TE/TM part is given by

$$
\begin{aligned}
\left\langle\mathbf{j}^{\mathrm{TE} / \mathrm{TM}}\right\rangle=\frac{c \varepsilon}{2 \omega^{2}} \operatorname{Re}\left\{i\left(k^{2} c_{\mathrm{TE}} c_{\mathrm{TM}}^{*}+c_{\mathrm{TE}}^{*} c_{\mathrm{TM}} k_{z}^{2}\right)\left(\nabla_{\mathrm{T}} \varphi^{*} \times \nabla_{\mathrm{T}} \varphi\right) \cdot\left(\mathbf{r} \times \hat{\mathbf{e}}_{3}\right)\right. \\
\left.+c_{\mathrm{TE}}^{*} c_{\mathrm{TM}} k_{z} k_{\mathrm{T}}^{2} \mathbf{r} \times\left[\hat{\mathbf{e}}_{3} \times \nabla_{\mathrm{T}}\left(\varphi^{*} \varphi\right)\right]\right\}
\end{aligned}
$$

The set of Eqs. (16), (17) and (18) provide a useful simple recipe to calculate the orbital angular momentum of any invariant beam in terms of scalar potentials. It is important to remark that Eq. (18) physically represents the orbital angular momentum propagation due to the superposition of modes TE/TM.

\subsection{Example: orbital angular momentum for Bessel beams using a scalar potential}

The properties of the orbital angular momentum have been extensively investigated by different means $[\underline{3}, \underline{50}]$. However, to the best of our knowledge, the study of the orbital angular momentum arising from mode superposition has not been attempted before; nevertheless, the interference Bessel beams have been applied in the micro-manipulation of particles $[\underline{51}, \underline{52}]$. With this in mind, and as an illustrative example, we analyze the particular case of the orbital angular momentum of Bessel beams. 
The transversal solution of the scalar Helmholtz equation in cylindrical coordinates is

$$
\varphi(r, \theta)=J_{m}\left(k_{\mathrm{T}} r\right) \exp (\operatorname{im} \theta)
$$

where $m$ is any integer, $J_{m}(\zeta)$ is the Bessel function of the first kind of order $m$, and $k_{\mathrm{T}}=k \sin \beta$ is the transversal vector [16]. Substituting (19) in (16), (17) and (18), we obtain the orbital angular momentum of a Bessel beam. The radial angular momentum is

$$
\begin{aligned}
\left\langle j_{r}\right\rangle=\frac{c \varepsilon}{2 \omega^{2}} \frac{z k_{\mathrm{T}}^{2}}{r}\{ & -\left(\left|c_{\mathrm{TE}}\right|^{2}+\left|c_{\mathrm{TM}}\right|^{2}\right) k m J_{m}^{2}\left(r k_{\mathrm{T}}\right) \\
& \left.+2 k_{z} \operatorname{Re}\left(c_{\mathrm{TE}}^{*} c_{\mathrm{TM}}\right) J_{m}\left(r k_{\mathrm{T}}\right)\left[r k_{\mathrm{T}} J_{m-1}\left(r k_{\mathrm{T}}\right)-m J_{m}\left(r k_{\mathrm{T}}\right)\right]\right\}
\end{aligned}
$$

while the azimuthal angular momentum component is

$$
\begin{aligned}
\left\langle j_{\theta}\right\rangle= & \frac{\varepsilon}{2 r \omega}\left\{-\left(\left|c_{\mathrm{TE}}\right|^{2}+\left|c_{\mathrm{TM}}\right|^{2}\right) k_{z}\right. \\
& \times\left[r^{2} k_{\mathrm{T}}^{2} J_{m-1}^{2}\left(r k_{\mathrm{T}}\right)+2 m^{2} J_{m}^{2}\left(r k_{\mathrm{T}}\right)-2 m r k_{T} J_{m-1}\left(r k_{\mathrm{T}}\right) J_{m}\left(r k_{\mathrm{T}}\right)\right] \\
& \left.+2 k m J_{m}\left(r k_{\mathrm{T}}\right)\left[r k_{\mathrm{T}} J_{m-1}\left(r k_{\mathrm{T}}\right)-m J_{m}\left(r k_{\mathrm{T}}\right)\right] \operatorname{Re}\left(c_{\mathrm{TE}} c_{\mathrm{TM}}^{*}+c_{\mathrm{TE}}^{*} c_{\mathrm{TM}} \frac{k_{z}^{2}}{k^{2}}\right)\right\}
\end{aligned}
$$

and the longitudinal component is

$$
\begin{aligned}
\left\langle j_{z}\right\rangle=\frac{c \varepsilon}{2 \omega^{2}} k_{\mathrm{T}}^{2}\{ & \left(\left|c_{\mathrm{TE}}\right|^{2}+\left|c_{\mathrm{TM}}\right|^{2}\right) m k J_{m}^{2}\left(r k_{\mathrm{T}}\right) \\
& \left.+2 k_{z} \operatorname{Re}\left(c_{\mathrm{TE}}^{*} c_{\mathrm{TM}}\right) J_{m}\left(r k_{\mathrm{T}}\right)\left[r k_{\mathrm{T}} J_{m-1}\left(r k_{\mathrm{T}}\right)-m J_{m}\left(r k_{\mathrm{T}}\right)\right]\right\}
\end{aligned}
$$

As can be observed, all terms have a clear contribution of modes superposition, which in general is different from zero. Even, for the most simple case, when the azimuthal value is zero, $m=0$, the mixed modes are given by

$$
\begin{aligned}
& \left\langle j_{r}\right\rangle=\operatorname{Re}\left(c_{\mathrm{TE}}^{*} c_{\mathrm{TM}}\right) \frac{c \varepsilon}{\omega^{2}} k_{z} k_{\mathrm{T}}^{3} z J_{0}\left(r k_{\mathrm{T}}\right) J_{1}\left(r k_{\mathrm{T}}\right) \\
& \left\langle j_{\theta}\right\rangle=\left(\left|c_{\mathrm{TE}}\right|^{2}+\left|c_{\mathrm{TM}}\right|^{2}\right) \frac{\varepsilon}{2 \omega} k_{z} k_{\mathrm{T}}^{2} r J_{1}^{2}\left(r k_{\mathrm{T}}\right) \\
& \left\langle j_{z}\right\rangle=-\operatorname{Re}\left(c_{\mathrm{TE}}^{*} c_{\mathrm{TM}}\right) \frac{c \varepsilon}{\omega^{2}} k_{z} k_{\mathrm{T}}^{3} r J_{0}\left(r k_{\mathrm{T}}\right) J_{1}\left(r k_{\mathrm{T}}\right)
\end{aligned}
$$


If the mixing is considered, these results show that there is interference between the radial and azimuthal components. Writing $c_{\mathrm{TE}}=\left|c_{\mathrm{TE}}\right| \exp \left(i \varphi_{1}\right)$ and $c_{\mathrm{TM}}=\left|c_{\mathrm{TM}}\right| \exp \left(i \varphi_{2}\right)$, we have $\operatorname{Re}\left(c_{\mathrm{TE}}^{*} c_{\mathrm{TM}}\right)=\cos \left(\varphi_{1}-\varphi_{2}\right)$; thus this term contributes if there is a difference of phase between $c_{\mathrm{TE}}$ and $c_{\mathrm{TM}}$, otherwise this term is zero. Therefore, all the well-known results for the orbital angular momentum can be obtained.

\subsection{Longitudinal orbital angular momentum}

The above equations allow us to obtain the longitudinal orbital angular momentum for a particular transversal electric, magnetic or interference mode; the procedure is just to take the scalar product of (16), (17) and (32) with $\hat{\mathbf{e}}_{3}$. For any invariant beams the longitudinal orbital angular momentum can be obtained as

$$
\begin{aligned}
& \left\langle j_{z}^{\mathrm{TE}}\right\rangle=\frac{\varepsilon k_{\mathrm{T}}^{2}}{2 \omega}\left|c_{\mathrm{TE}}\right|^{2} \operatorname{Re}\left[\left(\varphi^{*} \mathcal{L}_{\mathrm{T}} \varphi\right) \cdot \hat{\mathbf{e}}_{3}\right] \\
& \left\langle j_{z}^{\mathrm{TM}}\right\rangle=-\frac{\varepsilon k_{\mathrm{T}}^{2}}{2 \omega}\left|c_{\mathrm{TM}}\right|^{2} \operatorname{Re}\left[\left(\varphi \mathcal{L}_{\mathrm{T}} \varphi^{*}\right) \cdot \hat{\mathbf{e}}_{3}\right] \\
& \left\langle j_{z}^{\mathrm{TE} / \mathrm{TM}}\right\rangle=\frac{\varepsilon}{2 \omega} \frac{k_{z}}{k} k_{\mathrm{T}}^{2} \operatorname{Re}\left[c_{\mathrm{TE}}^{*} c_{\mathrm{TM}}\left(\mathcal{L}_{\mathrm{T}}^{\perp}|\varphi|^{2}\right) \cdot \hat{\mathbf{e}}_{3}\right]
\end{aligned}
$$

where $\mathcal{L}_{\mathrm{T}}=-i \mathbf{r} \times \nabla_{\mathrm{T}}$ and $\mathcal{L}_{\mathrm{T}}^{\perp}=\mathbf{r} \times \nabla_{\mathrm{T}}^{\perp}$. Where $\nabla_{\mathrm{T}}^{\perp}$ is given by Eq. (8) and which physically means a $\pi / 2$ rotation of $\nabla_{\mathrm{T}}$. Note that only the transversal structure beam, which is given for a single scalar potential $\varphi$, is required; all terms are proportional to $k_{\mathrm{T}}^{2}$. Equation (28) is given in terms of mixed modes and it is proportional to the ratio $k_{z} / k$. In the particular case of the Bessel beams, we substitute expression (19) into (26) and (27) obtaining the following results

$$
\left\langle j_{z}^{\mathrm{TE}}\right\rangle=\frac{\varepsilon k_{\mathrm{T}}^{2}}{2 \omega}\left|c_{\mathrm{TE}}\right|^{2} m J_{m}^{2}\left(r k_{\mathrm{T}}\right)
$$

and

$$
\left\langle j_{z}^{\mathrm{TM}}\right\rangle=\frac{\varepsilon k_{\mathrm{T}}^{2}}{2 \omega}\left|c_{\mathrm{TM}}\right|^{2} m J_{m}^{2}\left(r k_{\mathrm{T}}\right)
$$

We have obtained a well-known defined orbital angular momentum; these results recover the results reported in [료] for Bessel beams. A well-defined longitudinal orbital angular momentum is obtained for TE and TM modes when $m=0$, since there is no orbital angular momentum in the direction of propagation; if $m \neq 0$, the beam carries orbital angular momentum in the direction of propagation [ $\underline{2}, \underline{3}]$. Additionally, using the Eq. (29), Eq. (30) and the longitudinal electromagnetic energy density reported in [29], 
it is straightforward to verify that the ratio of $\left\langle j_{z}^{\mathrm{TE}}\right\rangle /\langle U\rangle_{z}=\left\langle j_{z}^{\mathrm{TM}}\right\rangle /\langle U\rangle_{z} \propto m / \omega[\underline{28}]$. These results confirm a well-defined values of angular momentum and energy for this nonparaxial approximation.

For the case of longitudinal mixed modes, substituting (19) into (28) and after some algebraic manipulation, we get

$$
\left\langle j_{z}^{\mathrm{TE} / \mathrm{TM}}\right\rangle=\frac{\varepsilon}{\omega} \frac{k_{z}}{k} k_{\mathrm{T}}^{2} \operatorname{Re}\left(c_{\mathrm{TE}}^{*} c_{\mathrm{TM}}\right)\left\{\frac{\mathrm{d}}{\mathrm{d} r}\left[r J_{m}^{2}\left(r k_{\mathrm{T}}\right)\right]-J_{m}^{2}\left(r k_{\mathrm{T}}\right)\right\}
$$

In this more general case, the longitudinal orbital angular momentum is not proportional to the topological charge $m$, as in the single mode case. Now it is related to the radial derivative of the square field weighted by its radius minus the intensity of the incident field.

In Fig. 1, it is shown the transverse electric $\left|\left\langle j_{z}^{\mathrm{TE}}\right\rangle\right|$ and the transverse magnetic $\left|\left\langle j_{z}^{\mathrm{TM}}\right\rangle\right|$ longitudinal orbital angular momentum for Bessel beams with $m=1$ and $m=2$.

In Fig. 2 the mixed modes superposition $\left|\left\langle j_{z}^{\mathrm{TE} / \mathrm{TM}}\right\rangle\right|$ longitudinal orbital angular momentum is shown for $m=1$ and for $m=2$. We obtain a well-defined regions due to the interference superposition modes which can be observed. This feature can be useful; the application of Bessel beams for optical manipulation has been proposed [1] , and it has recently been successfully developed for the case of a single-beam or counter-propagating beam trapping [53].

Recently, in [54], it has been shown how combining two Bessel beams, as in Eq. (19), with topological charges $m= \pm 1$, makes possible to generate a Hermite-Gauss (HG) beam, which possesses a well-defined orbital angular momentum [55]. Moreover, it is

a

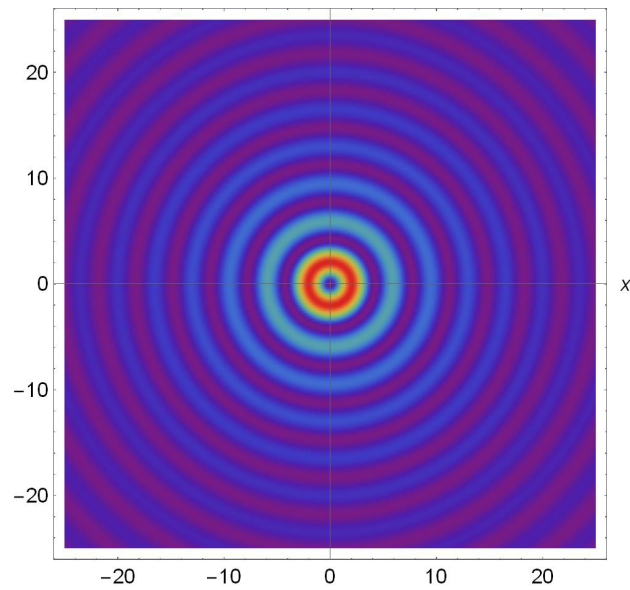

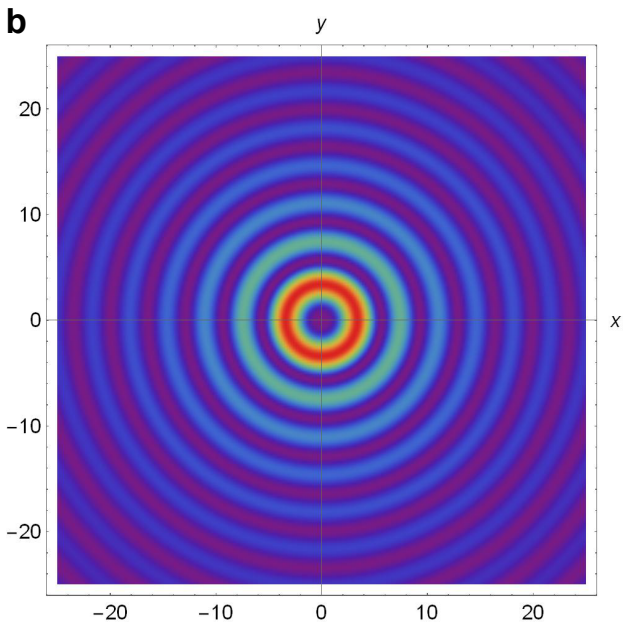

Fig. 1. Longitudinal orbital angular momentum $\left|\left\langle j_{z}^{\mathrm{TE}}\right\rangle\right|$ of a transversal electric Bessel beam with $m=1$ (a) and of a transversal magnetic $\left\langle j_{z}^{\mathrm{TM}}\right\rangle \mid$ with $m=2(\mathbf{b})$. 
a

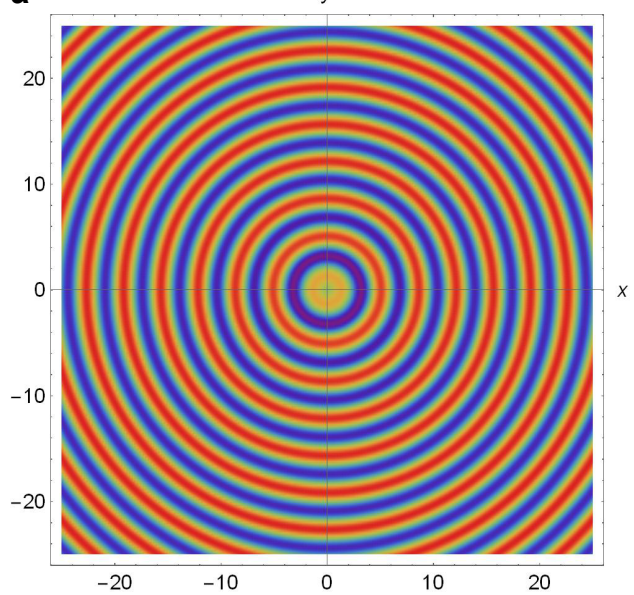

b

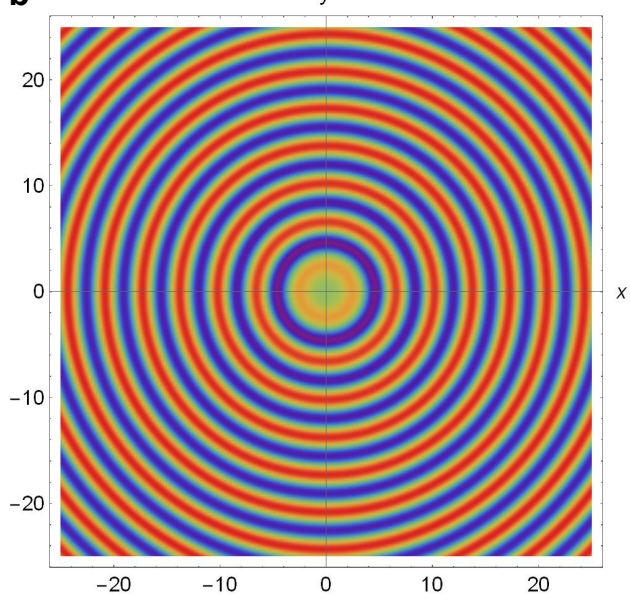

Fig. 2. The longitudinal orbital angular momentum interference term $\left|\left\langle j_{z}^{\mathrm{TE} / \mathrm{TM}}\right\rangle\right|$ of a Bessel beam, with $m=1$ (a), and with $m=2(\mathbf{b})$.

important to mention that a superposition of Hermite-Gauss beams can be transformed into a Laguerre-Gauss beam [56] which possesses a very well-known and characteristic $\exp (\operatorname{im} \varphi)$ factor [ $\underline{1}-\underline{3}]$. Thus, our generalized analytical formulation makes possible the study of the orbital angular momentum using a single scalar potential.

\subsection{The transversal interference term carry orbital angular momentum}

We can finally consider the paraxial approximation; i.e., the case when $k \approx k_{z}$. We substitute that approximation into Eq. (32), in which the second term vanishes. Without loss of generality, we can rewrite the complex constants defined above as $c_{\mathrm{TE}} \equiv i \alpha$ and $c_{\mathrm{TM}} \equiv \beta$. Then, it is straightforward to obtain

$$
\begin{aligned}
\left\langle\mathbf{j}^{\mathrm{TE} / \mathrm{TM}}\right\rangle=\frac{c \varepsilon}{2 \omega^{2}} \operatorname{Re}\{ & i\left(k^{2} c_{\mathrm{TE}} c_{\mathrm{TM}}^{*}+c_{\mathrm{TE}}^{*} c_{\mathrm{TM}} k_{z}^{2}\right)\left(\nabla_{\mathrm{T}} \varphi^{*} \times \nabla_{\mathrm{T}} \varphi\right) \cdot\left(\mathbf{r} \times \hat{\mathbf{e}}_{3}\right) \\
& \left.+c_{\mathrm{TE}}^{*} c_{\mathrm{TM}} k_{z} k_{\mathrm{T}}^{2} \mathbf{r} \times\left[\hat{\mathbf{e}}_{3} \times \nabla_{\mathrm{T}}\left(\varphi^{*} \varphi\right)\right]\right\}
\end{aligned}
$$

and

$$
\left\langle\mathbf{j}^{\mathrm{TE} / \mathrm{TM}}\right\rangle=\frac{\varepsilon}{2 c} \operatorname{Re}\left\{\left(\alpha^{*} \beta-\alpha \beta^{*}\right)\left(\nabla_{\mathrm{T}} \varphi \cdot \nabla_{\mathrm{T}}^{\perp} \varphi^{*}\right)\left(\hat{\mathbf{e}}_{1} u_{2}-\hat{\mathbf{e}}_{2} u_{1}\right)\right\}
$$

This expression clearly shows the vectorial transversal structure propagating due to mixed modes for any invariant beam. It is worth noticing the presence of the term $\sigma \equiv\left(\alpha^{*} \beta-\alpha \beta^{*}\right)$, which is due to the fact that any invariant beam can be generated as 
a superposition of plane waves [21]. This parameter $\sigma$ has been physically linked with polarization states and it has been usually reported in paraxial and nonparaxial approximations [2, 3] . After substituting a Bessel beam expressed by (19) into (33) and changing into cylindrical coordinates $x=r \cos \theta$ and $y=r \sin \theta$, we obtain

$$
\left\langle\mathbf{j}^{\mathrm{TE} / \mathrm{TM}}\right\rangle=m \frac{\varepsilon}{2 c} i \sigma k_{\mathrm{T}} J_{m}\left(r k_{\mathrm{T}}\right)\left[J_{m+1}\left(r k_{\mathrm{T}}\right)-J_{m-1}\left(r k_{\mathrm{T}}\right)\right]\left(\hat{\mathbf{e}}_{r} \sin \theta-\hat{\mathbf{e}}_{\theta} \cos \theta\right)
$$

This expression reveals that the orbital angular momentum contribution of the mixed modes is proportional to the topological azimuthal $m$ factor and depends linearly on the transversal wave vector. Taking $\sigma=0$ means linear polarization and the lack of possible interference modes; otherwise, for $\sigma= \pm i$, we have circular polarization with the existence of mixed modes.

Figure 3 shows the transverse amplitude vector with the mixed orbital angular momentum modes given by Eq. (34), for different values of $m$, with $\sigma=i$.

In Fig. 4, we change the azimuthal order to $m=2$ and the polarization is $\sigma=-i$. In both cases the intensity exhibits an azimuthally asymmetric shape which becomes circularly symmetrical.

Lastly, Eq. (34) can be written using the following Bessel function identity $J_{m-1}(z)$ $-J_{m+1}(z)=2 J_{m}^{\prime}(z)[\underline{57}]$, to obtain

$$
\begin{aligned}
\left\langle\mathbf{j}^{\mathrm{TE} / \mathrm{TM}}\right\rangle & =-i \frac{\varepsilon}{c} \sigma m k_{\mathrm{T}} J_{m}\left(k_{\mathrm{T}} r\right) J_{m}^{\prime}\left(k_{\mathrm{T}} r\right)\left(\hat{\mathbf{e}}_{r} \sin \theta-\hat{\mathbf{e}}_{\theta} \cos \theta\right) \\
& =-i \frac{\varepsilon}{2 c} \sigma m \frac{\mathrm{d} J_{m}^{2}\left(k_{\mathrm{T}} r\right)}{\mathrm{d} r}\left(\hat{\mathbf{e}}_{r} \sin \theta-\hat{\mathbf{e}}_{\theta} \cos \theta\right)
\end{aligned}
$$

a

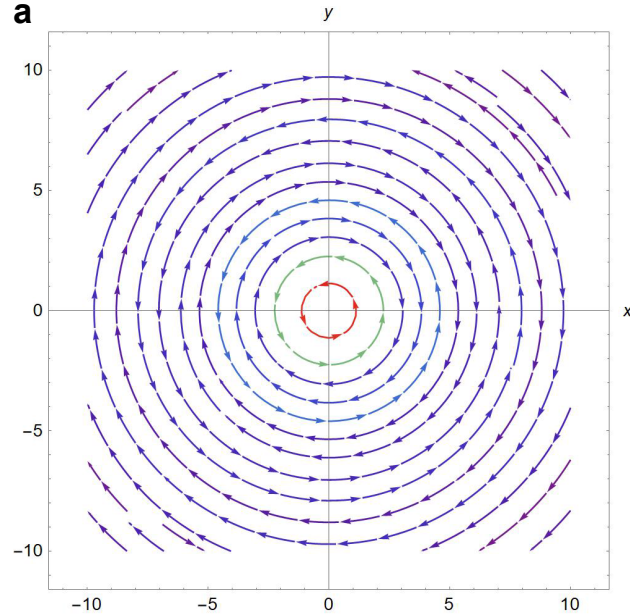

b

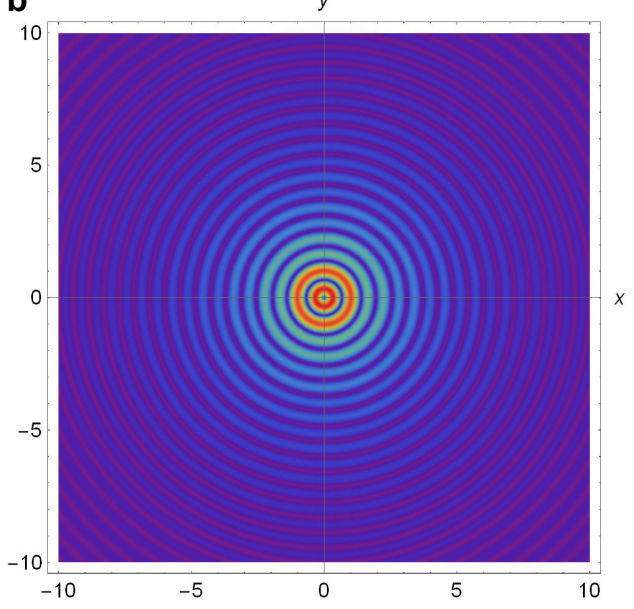

Fig. 3. The transversal vector orbital angular momentum (a) and its intensity (b) for a Bessel beam with $m=1$ and $\sigma=i$. 
a

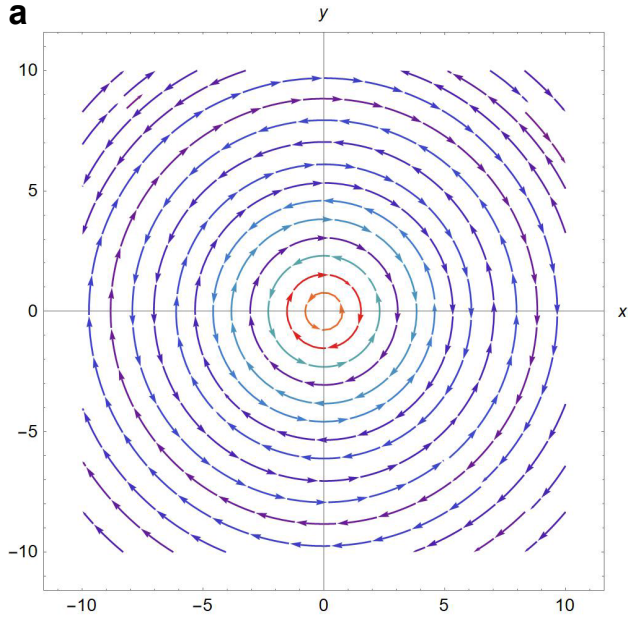

b

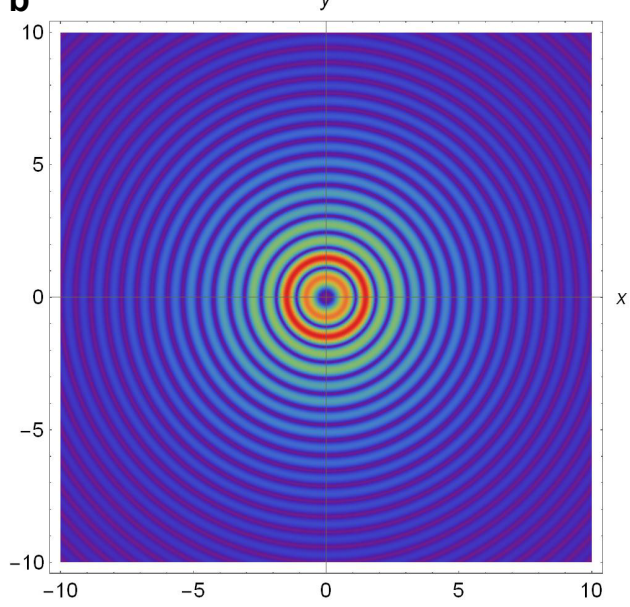

Fig. 4. The transversal vector orbital angular momentum (a) and its intensity (b) for a Bessel beam with $m=2$ and $\sigma=-i$.

It is possible to identify the input field intensity $I(r)$ as $I(r)=|\mathbf{E}|^{2}=J_{m}^{2}\left(k_{\mathrm{T}} r\right)$, and using $c=\omega / k$, we get

$$
\left\langle\mathbf{j}^{\mathrm{TE} / \mathrm{TM}}\right\rangle=-i \frac{\varepsilon \sigma}{2} m \frac{k}{\omega} \frac{\mathrm{d} I(r)}{\mathrm{d} r}\left(\hat{\mathbf{e}}_{r} \sin \theta-\hat{\mathbf{e}}_{\theta} \cos \theta\right)
$$

Thus, the transversal mixed modes orbital angular momentum can be calculated as the radial derivative of the input field intensity with a well-defined topological charge. Notice also that this expression contains the optical parameter, and it is proportional to the topological charge $m$. It is worth mentioning that a similar term was

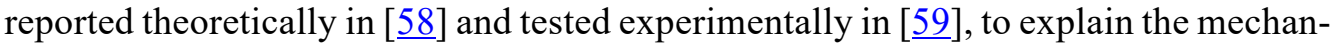
ical action of the spin part of the internal energy flow. There, the authors also showed the possibility of controllable motion of suspended particles by changing the polarization of the input field.

\section{Conclusions}

We have investigated the transversal and longitudinal propagation of orbital angular momentum for invariant beams using a single scalar potential. We have proved that the invariant beams satisfy Maxwell equations and possess a well-defined orbital angular momentum [28]. We have shown that the superposition of non-paraxial vectorial beams with axial symmetry can provide a well-defined orbital angular momentum. These results exhibit how the modes superposition affects the angular momentum flux density and causes reverse propagation in the case of the fractional Bessel beams $[\underline{60}, \underline{61}]$. In $[\underline{62}]$, the authors have studied the importance to handle the amplitude, phase and polarization 
in order to design structured fields to study the spin-orbit interactions in Bessel beams. Adopting the single scalar potential approach, presented here, may be useful to find interesting applications, as the electromagnetic spin and canonical momentum for paraxial and non-paraxial beams recently reported in [흐 $\underline{65}]$.

Acknowledgment - This research was supported by Basic Science Research Program through the National Research Foundation of Korea (NRF) funded by the Ministry of Science and ICT (NRF2017R1E1A1A01077717).

\section{References}

[1] Allen L., Beijersbergen M.W., Spreeuw R.J.C., Woerdman J.P., Orbital angular momentum of light and the transformation of Laguerre-Gaussian laser modes, Physical Review A 45(11), 1992, pp. 8185-1889, DOI: 10.1103/PhysRevA.45.8185.

[2] Yao A.M., PAdgett M.J., Orbital angular momentum: origins, behavior and applications, Advances in Optics and Photonics 3(2), 2011, pp. 161-204, DOI: 10.1364/AOP.3.000161.

[3] Padgett M.J., Orbital angular momentum 25 years on, Optics Express 25(10), 2017, pp. 11265-11274, DOI: 10.1364/OE.25.011265.

[4] Kerber R.M., Fitzgerald J.M., Reiter D.E., OH S.S., Hess O., Reading the orbital angular momentum of light using plasmonic nanoantennas, ACS Photonics 4(4), 2017, pp. 891-896, DOI: 10.1021/ acsphotonics.6b00980.

[5] Willner A.E., Huang H., Yan Y., Ren Y., Ahmed N., Xie G., Bao C., Li L., Cao Y., Zhao Z., Wang J., Lavery M.P.J., Tur M., Ramachandran S., Molisch A.F., Ashrafi N., Ashrafi S., Optical communications using orbital angular momentum beams, Advances in Optics and Photonics 7(1), 2015, pp. 66-106, DOI: 10.1364/AOP.7.000066.

[6] Volke-Sepulveda K., Ley-Koo E., General construction and connections of vector propagation invariant optical fields: TE and TM modes and polarization states, Journal of Optics A: Pure and Applied Optics 8(10), 2006, pp. 867-877, DOI: 10.1088/1464-4258/8/10/008.

[7] Volke-Sepulveda K., Garces-Chavez S., Chavez-Cerda S., Arlt J., Dholakia K., Orbital angular momentum of a high-order Bessel light beam, Journal of Optics B: Quantum and Semiclassical Optics 4(2), 2002, pp. S82-S89, DOI: 10.1088/1464-4266/4/2/373.

[8] Flores-Pérez A., Hernández-Hernández J., Jáuregui R., Volke-Sepúlveda K., Experimental generation and analysis of first-order TE and TM Bessel modes in free space, Optics Letters 31(11), 2006, pp. 1732-1734, DOI: 10.1364/OL.31.001732.

[9] Marston P.L., Scattering of a Bessel beam by a sphere, Journal of the Acoustical Society of America 121(2), 2007, pp. 753-758, DOI: $10.1121 / 1.2404931$.

[10] Marston P.L., Scattering of a Bessel beam by a sphere: II. Helicoidal case and spherical shell example, Journal of the Acoustical Society of America 124(5), 2008, pp. 2905-2910, DOI: 10.1121/1.2973230.

[11] Belafhal A., Chafiq A., Hricha Z., Scattering of Mathieu beams by a rigid sphere, Optics Communications 284(12), 2011, pp. 3030-3035, DOI: 10.1016/j.optcom.2011.02.021.

[12] Belafhal A., Ez-Zariy L., ChafiQ A., NeBdi H., Analysis of the scattering far field of a nondiffracting parabolic beam by a rigid sphere, Physical and Chemical News 60, 2011, pp. 15-21.

[13] Shoorian H., Scattering of linearly polarized Bessel beams by dielectric spheres, Journal of Quantitative Spectroscopy and Radiative Transfer 199, 2017, pp. 12-19, DOI: 10.1016/j.jqsrt.2017.05.020.

[14] Rondón-Ojeda I., Soto-Eguíbar F., Generalized optical theorem for propagation invariant beams, Optik 137, 2017, pp. 17-24, DOI: 10.1016/j.ijleo.2017.02.069.

[15] RAJABI M., MoJAHED A., Acoustic radiation force control: pulsating spherical carriers, Ultrasonics 83, 2018, pp. 146-156, DOI: 10.1016/j.ultras.2017.06.002. 
[16] Hernandez-Figueroa H.E., Zamboni-Rached M., Recami E., Non-diffracting Waves, Wiley, 2013.

[17] DuRnin J., Exact solutions for nondiffracting beams. I. The scalar theory, Journal of the Optical Society of America A 4(4), 1987, pp. 651-654, DOI: 10.1364/JOSAA.4.000651.

[18] Gutiérrez-Vega J.C., Iturbe-Castillo M.D., Chávez-Cerda S., Alternative formulation for invariant optical fields: Mathieu beams, Optics Letters 25(20), 2000, pp. 1493-1495, DOI: 10.1364/ OL.25.001493.

[19] Bandres M.A., Gutiérrez-Vega J.C., Chávez-Cerda S., Parabolic nondiffracting optical wave fields, Optics Letters 29(1), 2004, pp. 44-46, DOI: 10.1364/OL.29.000044.

[20] WhitTAKer E.T., On the partial differential equations of mathematical physics, Mathematische Annalen 57(3), 1903, pp. 333-355, DOI: 10.1007/BF01444290.

[21] Nieto Vesperinas M., Scattering and Diffraction in Physical Optics, Wiley, New York 1991.

[22] Bouchal Z., Nondiffracting optical beams: physical properties, experiments, and applications, Czechoslovak Journal of Physics 53(7), 2003, pp. 537-578, DOI: 10.1023/A:1024802801048.

[23] Fahrbach F.O., Rohrbach A., Propagation stability of self-reconstructing Bessel beams enables contrast-enhanced imaging in thick media, Nature Communications 3, 2012, article 632, DOI: 10.1038/ ncomms1646.

[24] Olarte O.E., Andilla J., Gualda E.J., Loza-Alvarez P., Light-sheet microscopy: a tutorial, Advances in Optics and Photonics 10(1), 2018, pp. 111-179, DOI: 10.1364/AOP.10.000111.

[25] Asoubar D., Zhang S., Wyrowski F., Kunn M., Parabasal field decomposition and its application to non-paraxial propagation, Optics Express 20(21), 2012, pp. 23502-23517, DOI: 10.1364/OE.20. $\underline{023502 .}$.

[26] Zhang S., Hellmann C., Wyrowski F., Algorithm for the propagation of electromagnetic fields through etalons and crystals, Applied Optics 56(15), 2017, pp. 4566-4576, DOI: $10.1364 / A O .56 .004566$.

[27] Novitsky A., Ding W., Wang M., Gao D., Lavrinenko A.V., Qiu C.W., Pulling cylindrical particles using a soft-nonparaxial tractor beam, Scientific Reports 7, 2017, article 652, DOI: $10.1038 / \mathrm{s} 41598$ -017-00735-2.

[28] BRANDAO P.A., Nonparaxial TE and TM vector beams with well-defined orbital angular momentum, Optics Letters 37(5), 2012, pp. 909-911, DOI: 10.1364/OL.37.000909.

[29] Rondon-Ojeda I., Soto-Eguibar F., Electromagnetic field theory for invariant beams using scalar potentials, Progress in Electromagnetic Research B 66, 2016, pp. 49-61, DOI: 10.2528/PIERB15123102.

[30] Novitsky A.V., Novitsky D.V., Negative propagation of vector Bessel beams, Journal of the Optical Society of America A 24(9), 2007, pp. 2844-2849, DOI: 10.1364/JOSAA.24.002844.

[31] Chen J., Ng J., Lin Z., Chan C.T., Optical pulling force, Nature Photonics 5, 2011, pp. 531-534, DOI: $10.1038 /$ nphoton.2011.153.

[32] Costa J.T., Silveirinha M.G., AlÙ A., Poynting vector negative-index metamaterials, Physical Review B 83(16), 2011, article 165120, DOI: 10.1103/PhysRevB.83.165120.

[33] Novitsky A., Qiu C.W., WANG H., Single gradientless light beam drags particles as tractor beams, Physical Review Letters 107(20), 2011, article 203601, DOI: 10.1103/PhysRevLett.107.203601.

[34] Qiu C.W., Palima D., Novitsky A., Gao D., Ding W., Zhukovsky S.V., Gluckstad J., Engineering light-matter interaction for emerging optical manipulation applications, Nanophotonics 3(3), 2014, pp. 181-201, DOI: 10.1515/nanoph-2013-0055.

[35] Salem M.A., Bagci H., Energy flow characteristics of vector X-Waves, Optics Express 19(9), 2011, pp. 8526-8532, DOI: 10.1364/OE.19.008526.

[36] Sukhov S., Dogariu A., On the concept of "tractor beams", Optics Letters 35(22), 2010, pp. 3847 -3849, DOI: $10.1364 /$ OL.35.003847.

[37] Rondon-Ojeda I., Soto-Eguibar F., Properties of the Poynting vector for invariant beams: negative propagation in Weber beams, Wave Motion 78, 2018, pp. 176-184, DOI: 10.1016/j.wavemoti.2018.02.003.

[38] Chen J., WAN C., ZhAN Q., Vectorial optical fields: recent advances and future prospects, Science Bulletin 63(1), 2018, pp. 54-74, DOI: 10.1016/j.scib.2017.12.014. 
[39] Garcia-Gracia H., Perez-Garcia B., Lopez-Mago D., Hernandez-Aranda R.I., Gutiérrez-Vega J.C., Measurement of orbital angular momentum with an off-axis superposition of vector modes, Journal of Optics 16(4), 2014, article 045702, DOI: 10.1088/2040-8978/16/4/045702.

[40] ORnigotti M., Conti C., SZAmeit A., Effect of orbital angular momentum on nondiffracting ultrashort optical pulses, Physical Review Letters 115(10), 2015, article 100401, DOI: 10.1103/PhysRevLett. $\underline{115.100401 .}$

[41] Bekshaev A.Y., Bliokh K.Y., Nori F., Transverse spin and momentum in two-wave interference, Physical Review X 5(1), 2015, article 011039, DOI: 10.1103/PhysRevX.5.011039.

[42] Li M., Yan S., Liang Y., Zhang P., Yao B., Spinning of particles in optical double-vortex beams, Journal of Optics 20(2), 2018, article 025401, DOI: 10.1088/2040-8986/aaa0e9.

[43] Litvin I.A., Dudley A., Forbes A., Poynting vector and orbital angular momentum density of superpositions of Bessel beams, Optics Express 19(18), 2011,pp. 16760-16771, DOI: 10.1364/OE.19.016760.

[44] FANG L., WANG J., Optical angular momentum derivation and evolution from vector field superposition, Optics Express 25(19), 2017, pp. 23364-23375, DOI: 10.1364/OE.25.023364.

[45] Stratton J.A., Electromagnetic Theory, McGraw-Hill, 1941.

[46] Boyer C.P., Kalnins E.G., Miller W., Symmetry and separation of variables for the Helmholtz and Laplace equations, Nagoya Mathematical Journal 60, 1976, pp. 35-80, DOI: $10.1017 /$ $\underline{\mathrm{S} 0027763000017165 .}$.

[47] JACKSON J.D., Classic Electrodynamics, Third Edition, Wiley, 1999.

[48] Bajer J., Horák R., Nondiffractive fields, Physical Review E 54(3), 1996, pp. 3052-3054, DOI: 10.1103/PhysRevE.54.3052.

[49] Milonni P.W., Boyd R.W., Momentum of light in a dielectric medium, Advances in Optics and Photonics 2(4), 2010, pp. 519-553, DOI: 10.1364/AOP.2.000519.

[50] Mendez G., Fernandez-VazQuez A., Paez Lopez R., Orbital angular momentum and highly efficient holographic generation of nondiffractive TE and TM vector beams, Optics Communications 334,

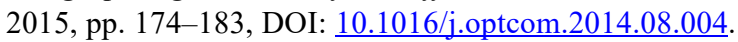

[51] McGloin D., Garcés-Chávez V., Dholakia, K., Interfering Bessel beams for optical micromanipulation, Optics Letters 28(8), 2003, pp. 657-659, DOI: 10.1364/OL.28.000657.

[52] Mazilu M., Stevenson D.J., Gunn-Moore F., Dholakia K., Light beats the spread: "non-diffracting” beams, Laser \& Photonics Reviews 4(4), 2010, pp. 529-547, DOI: 10.1002/lpor.200910019.

[53] ZнаO C., Practical guide to the realization of a convertible optical trapping system, Optics Express 25(3), 2017, pp. 2496-2510, DOI: 10.1364/OE.25.002496.

[54] Ornigotti M., Aiello A., Radially and azimuthally polarized nonparaxial Bessel beams made simple, Optics Express 21(13), 2013, pp. 15530-15537, DOI: 10.1364/OE.21.015530.

[55] Kotlyar V.V., Kovalev A.A., Hermite-Gaussian modal laser beams with orbital angular momentum, Journal of the Optical Society of America A 31(2), 2014, pp. 274-282, DOI: 10.1364/JOSAA.31.000274.

[56] Wang Y., Chen Y., Zhang Y., Chen H., Yu S., Generalised Hermite-Gaussian beams and mode transformations, Journal of Optics 18(5), 2016, article 055001, DOI: 10.1088/2040-8978/18/5/055001.

[57] Abramowitz M., Handbook of Mathematical Functions, Dover Publications, New York 1974.

[58] Angelsky O.V., Bekshaev A.Y., Maksimyak P.P., Maksimyak A.P., Hanson S.G., Zenkova C.Y., Orbital rotation without orbital angular momentum: mechanical action of the spin part of the internal energy flow in light beams, Optics Express 20(4), 2012, pp. 3563-3571, DOI: 10.1364/OE.20.003563.

[59] Angelsky O.V., Bekshaev A.Y., Maksimyak P.P., Maksimyak A.P., Mokhun I.I., Hanson S.G., Zenkova C.Y., Tyurin A.V., Circular motion of particles suspended in a Gaussian beam with circular polarization validates the spin part of the internal energy flow, Optics Express 20(10), 2012, pp. 11351-11356, DOI: 10.1364/OE.20.011351.

[60] Mitri F.G., Superposition of nonparaxial vectorial complex-source spherically focused beams: axial Poynting singularity and reverse propagation, Physical Review A 94(2), 2016, article 023801, DOI: 10.1103/PhysRevA.94.023801. 
[61] MitRI F.G., Reverse propagation and negative angular momentum density flux of an optical nondiffracting nonparaxial fractional Bessel vortex beam of progressive waves, Journal of the Optical Society of America A 33(9), 2016, pp. 1661-1667, DOI: 10.1364/JOSAA.33.001661.

[62] Aleksanyan A., Brasselet E., Spin-orbit photonic interaction engineering of Bessel beams, Optica 3(2), 2016, pp. 167-174, DOI: 10.1364/OPTICA.3.000167.

[63] Bliokн K.Y., Nori F., Transverse and longitudinal angular momenta of light, Physics Reports 592, 2015, pp. 1-38, DOI: 10.1016/j.physrep.2015.06.003.

[64] Bliokh K.Y., Alonso M.A., Ostrovskaya E.A., Aiello A., Angular momenta and spin-orbit interaction of nonparaxial light in free space, Physical Review A 82(6), 2010, article 063825, DOI: 10.1103/PhysRevA.82.063825.

[65] Bliokh K.Y., Ivanov I.P., Guzzinati G., Clark L., Van Boxem R., Béché A., Juchtmans R., Alonso M.A., Schattschneider P., Nori F., Verbeeck J., Theory and applications of free-electron vortex states, Physics Reports 690, 2017, pp. 1-70, DOI: 10.1016/j.physrep.2017.05.006.

Received January 23, 2020

in revised form April 16, 2020 